\title{
Epidermal growth factor receptor and human epidermal growth receptor 2 expression in parotid mucoepidermoid carcinoma: Possible implications for targeted therapy
}

\author{
JINBIAO SHANG ${ }^{1,2}$, YONGJIE SHUI ${ }^{3}$, LIMING SHENG $^{3}$, KEJING WANG $^{2}$, \\ QIONGGE HU ${ }^{1}$ and QICHUN WEI ${ }^{1,3}$
}

\author{
${ }^{1}$ Department of Radiation Oncology, the Second Affiliated Hospital, Zhejiang University \\ School of Medicine, Hangzhou 310009; ${ }^{2}$ Department of Head and Neck Surgery, Zhejiang Cancer \\ Hospital, Hangzhou 310016; ${ }^{3}$ Cancer Institute, Zhejiang University, Hangzhou 310009, P.R. China
}

Received July 24, 2007; Accepted October 8, 2007

\begin{abstract}
The expression of epidermal growth factor receptor (EGFR) and human epidermal growth factor receptor 2 (HER2) was analyzed in immunohistochemical preparations from 46 primary parotid mucoepidermoid carcinomas (MEC). For the cases with lymph node metastases, the receptor expressions were investigated in parallel samples, primary tumour and metastasis, from each patient $(\mathrm{n}=11)$. The goal was to evaluate whether any of these receptors are suitable as a target for radionuclide-based imaging and therapy. The HercepTest scoring was used for the analysis of both HER2 and EGFR expression $(0,1+, 2+$ or $3+)$. EGFR overexpression $(2+/ 3+)$ was found in $67.4 \%(31 / 46)$ of the primary tumours. Out of the 11 cases with evaluated paired samples, EGFR overexpression was observed in $81.8 \%$ (9/11) of the primary tumours and $72.7 \%(8 / 11)$ of the corresponding lymph node metastases. There was only one patient who had EGFR overexpression in the primary tumours which changed to negative in the lymph node metastases but no changes occurred reciprocally. The HER2 overexpression was only found in $4.3 \%(2 / 46)$ of the primary mucoepidermoid carcinoma and none of the lymph node metastases $(0 / 11)$. EGFR and HER2 stainings were mainly found in the cell membranes. It was concluded that the majority of parotid mucoepidermoid carcinomas express EGFR strongly in their cell membranes and that lymph node metastases generally express EGFR to approximately the same extent as in the primary tumours. The stability in the EGFR expression is encouraging in the effort to develop radionuclide-based
\end{abstract}

Correspondence to: Dr Qichun Wei, Department of Radiation Oncology, the Second Affiliated Hospital, Cancer Institute, Zhejiang University School of Medicine, Jiefang Road 88, Hangzhou 310009, P.R. China

E-mail: qichun_wei@zju.edu.cn

Key words: mucoepidermoid carcinoma, clinical, epidermal growth factor receptor, human epidermal growth factor receptor 2, metastasis, overexpression of receptors
EGFR imaging agents. It is also possible that EGFR targeting agents (e.g. Iressa, Tarceva, Erbitux or radiolabelled antibodies) can be applied for the therapy of mucoepidermoid carcinoma.

\section{Introduction}

Mucoepidermoid carcinoma (MEC) is considered the most common type of salivary gland malignancy accounting for approximately one third of all salivary malignancies (1-4) and the most common malignant tumour of the parotid gland (5). Surgical resection has traditionally been considered the primary treatment at initial diagnosis, whereas radiotherapy is recommended for patients with poor prognostic features such as late-stage tumour, anaplastic histology, perineural invasion or inadequate margins $(6,7)$. Chemotherapy has been reserved for palliation of a minority of MEC patients with advanced, unresectable or metastatic disease and its role continues to evolve (7). The prognosis of MEC is mainly influenced by the histologic grade and the clinical stage. A tumour with a low grade of malignancy and an early clinical stage has a very low recurrence rate and high survival. In contrast, high grade tumours usually have a worse clinical outcome, with 5-year survival of around $30 \%(5,8,9)$. Distant metastasis is not uncommon in high-grade mucoepidermoid carcinoma of the parotid gland. According to Spiro et al (10), 35\% of high grade MEC in major salivary glands developed distant metastasis, and a higher incidence $(67 \%)$ of distant metastasis was reported by Emerick et al (5). Those presenting with recurrent or metastatic disease have limited treatment options. Therefore, there is a definite need for additional therapeutic strategies to improve the survival and quality of life for these patients. One strategy is molecular-targeted therapy, which is currently having a positive impact on the daily practice of clinical oncology. Another strategy is receptor-mediated tumour-targeting radionuclide therapy (11), which is based on the delivery of therapeutically relevant radionuclides directly to disseminated tumour cells, hopefully with minimal damage to normal tissues.

Epidermal growth factor receptor (EGFR) signaling pathways control a wide variety of cellular processes, 
including cellular proliferation, apoptosis and angiogenesis. Consequently, EGFR is one of the most-studied ligandreceptor systems and specific EGFR inhibition approaches are currently among the most promising and advanced in the clinical setting. Nowadays, EGFR-targeted drugs, including small-molecule tyrosine kinase inhibitors (e.g. Iressa and Tarceva) $(12,13)$, as well as the chimeric monoclonal antibody Cetuximab (Erbitux) (14), are among the most advanced anti-EGFR drugs at the clinical level. Recently, randomized clinical trials combining Cetuximab with radiotherapy have shown promising locoregional control and a reduction in mortality for patients with stage III-IV head and neck squamous cell carcinoma (15) and a better overall response rate was observed in a phase-III evaluation of Cetuximab in combination with cisplatin in recurrent/ metastatic head and neck cancer (16). Meanwhile, trastuzumab (Herceptin), a humanized monoclonal antibody which specifically targets the extracellular domain of HER2 (human epidermal growth factor receptor 2), was reported to have significant therapeutic effects in patients with HER2-positive metastatic breast cancer (17). So far, the literature on the trials of Herceptin in patients with advanced salivary carcinoma has been rare $(18,19)$. Haddad et al $(18)$ reported the results of weekly trastuzumab therapy, one of the three patients with mucoepidermoid carcinoma had a partial response that is still ongoing at 45 months.

The knowledge of EGFR levels within mucoepidermoid carcinoma was limited and the reported frequencies of EGFR overexpression varied greatly $(20,21)$. HER2 overexpression occurs in 0 to $38 \%$ of mucoepidermoid carcinoma (22-28). So far, no previous study has evaluated the EGFR and HER2 expression in metastases and compared such an expression with that of corresponding primary mucoepidermoid carcinoma. It is still unclear whether the metastases lose, gain or retain the receptor status relative to the primary tumours. For a receptor to be of interest for targeting, a similar expression in both the primary tumours and the disseminated lesions are required. Investigation into the receptor status between metastases and the primary tumours will provide valuable information on whether these receptors are suitable as a target for diagnostic and/or therapeutic procedures. In the present study, the expression of EGFR and HER2 was investigated immunohistochemically in a series of 46 parotid mucoepidermoid carcinomas and 11 paired lymph node metastases.

\section{Materials and methods}

Patients and samples. Forty-one patients with parotid gland mucoepidermoid carcinoma who were diagnosed and treated in Zhejiang Cancer Hospital, China, between 1994 and 2006, were enrolled in the present study. All the patients were treated with surgical resection and cervical lymph node dissection was performed in 35 patients and lymph node metastases were found pathologically in 6 of the cases. Another 5 cases of parotid mucoepidermoid carcinoma with lymph node metastases were collected from the Second Affiliated Hospital, Zhejiang University School of Medicine. In total, 46 patients with high quality material were finally included in the study. Clinical information was obtained from the
Table I. Tumour and patient characteristics $(n=46)$.

Characteristics

Patients, n (\%)

Differentiation of MEC

Low

Moderate

High

T-stages

$\mathrm{T} 1$

$\mathrm{T} 2$

T3

$\mathrm{T} 4$

Pathologic nodal involvement

Gender

Male

Female

Medium age, years

Male

Female

MEC, mucoepidermoid carcinoma.

hospital records and included patient age, sex, location of the primary tumour, disease stage, histological grade and nodal involvement. The patient and tumour characteristics of the analyzed cases are shown in Table I.

Briefly, the tissues were fixed in $4 \%$ buffered formalin, processed and embedded in paraffin. Sections, $4-\mu \mathrm{m}$ thick, were then cut and deparaffinized in xylene and hydrated, through graded concentrations of ethanol, to distilled water.

EGFR-staining. EGFR was assessed by immunohistochemistry using a streptavidin-biotin complex technique as previously described (29). After deparaffinization of the sections, endogenous peroxidase was blocked in $0.3 \% \mathrm{H}_{2} \mathrm{O}_{2}$ in PBS for $20 \mathrm{~min}$. For antigen retrieval, the sections were submitted to high temperature and pressure with Tris-EDTA buffer ( $\mathrm{pH}$ 9.0) for $5 \mathrm{~min}$. The slides were preincubated in PBS for $10 \mathrm{~min}$. The primary mouse monoclonal antibody directed against the EGF receptor (clone 31G7, Zymed labs, South San Francisco, CA, USA) was diluted at 1:100 and incubated overnight at $4{ }^{\circ} \mathrm{C}$. The secondary biotinylated antibodies (goat anti-mouse from Dako, Glostrup, Denmark) and the peroxidase-labelled streptavidin-biotin complex (Dako) were diluted at 1:200 and incubated for $30 \mathrm{~min}$ at room temperature. All slides were developed in $0.05 \%$ diaminobenzidine (Sigma, St. Louis, MO, USA) for $5 \mathrm{~min}$ and counterstained in Harris hematoxylin (Sigma). Finally, the slides were dehydrated through graded alcohol to xylene and mounted in organic mounting medium.

HER2-staining. The HER2 immunohistochemical staining was made as previously described (29). After deparaffinization, the sections were incubated in methanol and hydrogen peroxide 
Table II. EGFR and HER2 expression for the analyzed primary parotid mucoepidermoid carcinoma $(n=46)$.

\begin{tabular}{lcccc}
\hline & \multicolumn{4}{c}{ Immunohistochemical scores } \\
& 0 & $1+$ & $2+$ & $3+$ \\
\hline EGFR & 6 & 9 & 21 & 10 \\
HER2 & 27 & 17 & 1 & 1 \\
\hline
\end{tabular}

The scoring was based on a scale where 0 corresponded to completely negative staining, $1+$ corresponded to faint perceptible staining of the tumour cell membranes, $2+$ corresponded to moderate staining of the entire tumour cell membranes and 3+ was strong circumferential staining of the entire tumour cell membranes creating a fishnet pattern.

Table III. EGFR-scores for the analyzed primary mucoepidermoid carcinoma and the corresponding lymph node metastases $(n=11)$.

\begin{tabular}{lcccc}
\hline \multirow{2}{*}{$\begin{array}{l}\text { Primary tumour } \\
\text { EGFR-scores }\end{array}$} & \multicolumn{4}{c}{ Lymph node metastasis EGFR-scores } \\
& 0 & $1+$ & $2+$ & $3+$ \\
\hline 0 & 1 & 1 & 0 & 0 \\
$1+$ & 0 & 0 & 0 & 0 \\
$2+$ & 1 & 0 & 3 & 1 \\
$3+$ & 0 & 0 & 1 & 3
\end{tabular}

The scoring was based on a scale where 0 corresponded to completely negative staining, $1+$ corresponded to faint perceptible staining of the tumour cell membranes, $2+$ corresponded to moderate staining of the entire tumour cell membranes and 3+ was strong circumferential staining of the entire tumour cell membranes creating a fishnet pattern.

for 30 min quenching endogenous peroxidase. Antigen retrieval was done in waterbath at $95-98^{\circ} \mathrm{C}, \mathrm{pH} 6.0$ for $40 \mathrm{~min}$. Thereafter the glasses were cooled at room temperature and then washed in distilled water. Immunohistochemical stainings were performed using the Elite ABC kit (Vectastain, Vector Laboratories, Burlingame, CA). Blocking serum was applied for $15 \mathrm{~min}$ and followed by incubation with rabbit anti-human c-erbB-2 oncoprotein (code No. A 0485, Dako) diluted at 1:350. Sections were then incubated with the biotinylated secondary antibody and were visualised by using the peroxidase substrate 3-amino-9-ethyl-carbazole (AEC) (Sigma A-5754) as a chromogen. Finally, the sections were counterstained with Mayer's hematoxylin and mounted with Aquamount (BDH Ltd, Poole, UK).

EGFR and HER2-scores. The HER2 expression was scored using the HercepTest scoring criterion. The HER2-score was based on a scale where 0 corresponded to tumour cells that were completely negative, $1+$ corresponded to faintly perceptible staining of the tumour cell membranes, $2+$ corresponded to moderate staining of the entire tumour cell membranes and 3+ was a strong circumferential staining of the entire tumour cell membranes creating a fishnet pattern. The Canadian and the Dako HercepTest guidelines (30) were applied, which require more than $10 \%$ of the tumour cells to be stained. Cytoplasmic staining was considered non-specific and was not included in the scoring. As positive controls we used in-house positive control tissue sections as well as positive control sections supplied by Dako. As negative controls we used normal tissues, which are expected not to express HER2 such as connective tissue seen in the same sections as the tumour cells. In the metastases sections, we used lymphocytes and the surrounding capsule of the lymph nodes as negative internal controls. The expression pattern of EGFR is quite similar to that of HER2 and the EGFR expression was thus evaluated using the same scoring criterion as for HER2. As EGFR positive controls we used in-house positive control skin tissue sections. As negative controls we used connective tissue seen in the same sections as the tumour cells. In the metastases sections, we used lymphocytes and the surrounding capsule of the lymph nodes as negative internal controls.

\section{Results}

Expression of EGFR. The EGFR scores for the analyzed 46 primary mucoepidermoid carcinoma are shown in Table II. EGFR overexpression (2+ or $3+$ ) was found in $67.4 \%$ (31/46) of the primary tumours, $1+$ staining in 9 cases and negative EGFR staining in the remaining 6 cases. Out of the 11 cases with evaluated paired samples, the EGFR overexpression $(2+$ or $3+)$ was observed in $81.8 \%(9 / 11)$ of the primary tumours and $72.7 \%(8 / 11)$ of the corresponding lymph node metastases. There was a good agreement between the primary tumours and the corresponding lymph node metastases in the majority of cases. The important results from the EGFRscore analyses of the paired samples are summarized in Table III. In total, four changes were observed. However, there was only one patient who had EGFR overexpression in the primary tumours which changed to negative in the lymph node metastases, but no patients who had 0 or $1+$ in the primary tumours and changed to $2+$ or $3+$ in the lymph node metastases. Examples of staining patterns for a primary tumour and the corresponding metastasis (both of which were scored as 3+) are shown in Fig. 1A and B.

Expression of HER2. Table II shows the HER2 scores for the analyzed primary mucoepidermoid carcinoma. Generally, HER2 had a lower expression as compared to EGFR. An overexpression (2+ or $3+$ ) of HER2 was only found in $4.3 \%$ (2/46) of the primary mucoepidermoid carcinoma (Fig. 2). Seventeen cases $(37.0 \%)$ had only faint perceptible staining of the tumour cell membranes (1+), while $27(58.7 \%)$ of the 46 cases had no HER2 staining at all. Out of the eleven cases of mucoepidermoid carcinoma with paired lymph node metastases, 5 cases had a 1+ HER2 score in the primary tumours and the same stainings $(1+)$ in the lymph node metastases were observed in 4 out of the 5 cases, one case had negative HER2 staining in the metastasis. The other six cases had no HER2 staining either in the primary tumour or in the corresponding lymph node metastases. In total, just one 

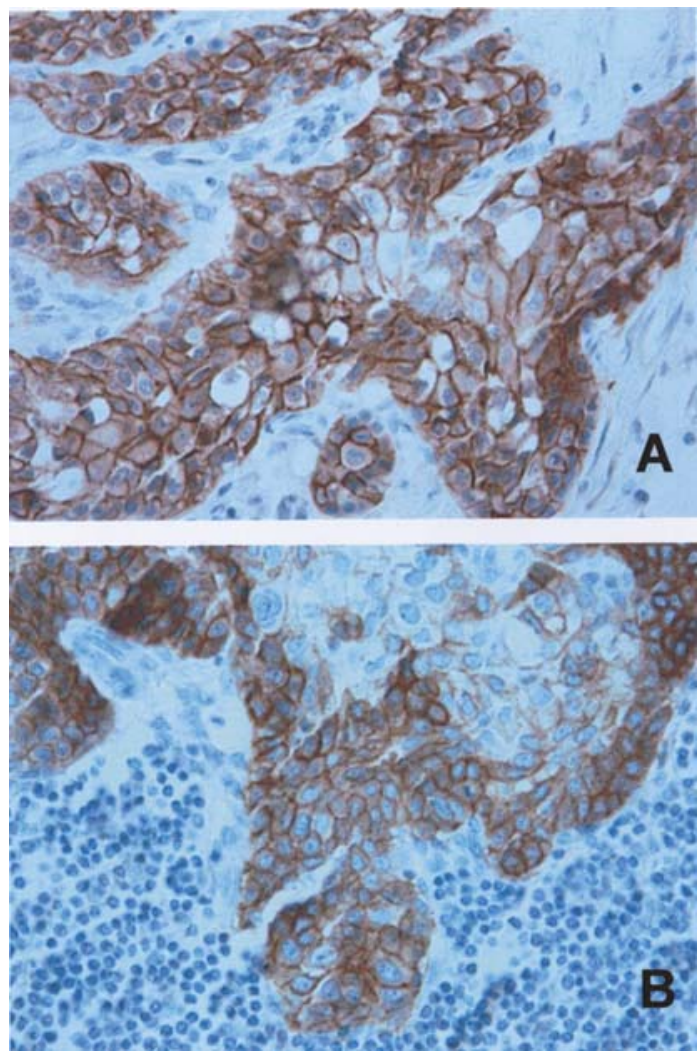

Figure 1. Comparisons of immunohistochemical brown EGFR staining of primary mucoepidermoid carcinoma (A) and corresponding metastases (B). Both $\mathrm{A}$ and $\mathrm{B}$ (from the same patient) were scored as $3+$. Original magnification $\mathrm{x} 40$.

change of HER2 scores between primary mucoepidermoid carcinoma and the corresponding lymph node metastases was observed.

\section{Discussion}

To our knowledge, the present study is the first to compare the EGFR and HER2 receptor status in primary mucoepidermoid carcinomas with their lymph node metastases. The goal was to evaluate whether any of these receptors are suitable as a target for clinical diagnosis and therapy, including radionuclide-based imaging and radiation therapy.

The expression of the epidermal growth factor receptor, EGFR, in mucoepidermoid carcinoma is not well-documented. According to the limited literature, the overexpression of EGFR was reported to be 25 and $77 \%$ respectively $(20,21)$. EGFR-targeted drugs are now commercially available, including small-molecule tyrosine kinase inhibitors (e.g. Iressa and Tarceva) $(12,13)$, as well as the chimeric monoclonal antibody cetuximab (Erbitux) (14). However, with the exception of a total of four cases reported in two meeting abstracts (ASCO), these EGFR-targeted drugs have yet to be tried for the therapy of mucoepidermoid carcinoma. It is, from previous studies, still an unanswered question as to whether the metastases lose, gain or retain EGFR status relative to the primary tumour. Studies on the EGFR status of the metastatic lymph node of mucoepidermoid carcinoma will provide precious knowledge to evaluate whether the

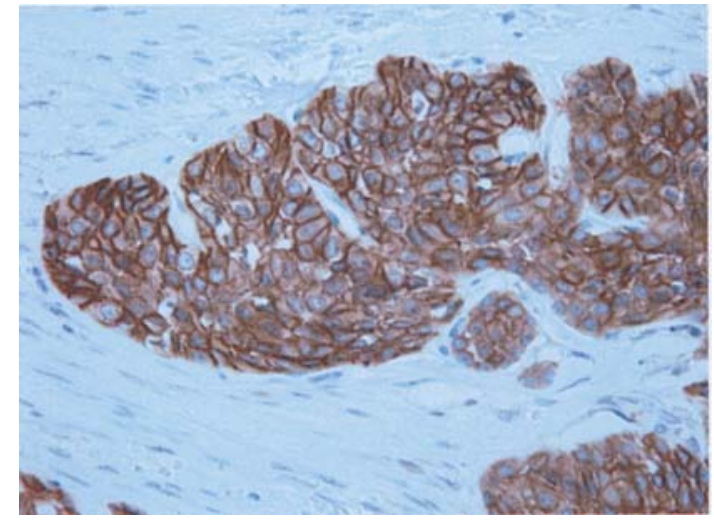

Figure 2. Example of immunohistochemical brown HER2 staining of primary mucoepidermoid carcinoma, scored as $3+$. This case had no pathological lymph node metastasis. Original magnification $\mathrm{x} 40$.

receptor is of interest for diagnostic and/or therapeutic procedures or not.

In the present study, the EGFR expression was identified in the primary mucoepidermoid carcinoma and, in eleven cases, the corresponding lymph node metastases as well. A high frequency of EGFR overexpression has been reported in head and neck cancers (31). The overexpression of EGFR is generally believed to be common in salivary gland carcinoma, although the incidence of positive EGFR staining was found to be $25 \%(2 / 8)$ in an earlier study with a small sample size (20). Gibbons et al found 17 out of $22(77 \%)$ mucoepidermoid carcinoma cases with EGFR overexpression (21). We reported on a series of 46 patients of mucoepidermoid carcinoma. It is the largest series considering that the EGFR status of mucoepidermoid carcinoma and EGFR overexpression $(2+/ 3+)$ was found in $67.4 \%$ of the cases. Our findings confirmed the result of a high EGFR overexpression in mucoepidermoid carcinoma found by Gibbons et al (21). Furthermore, we found that the frequency of the EGFR overexpression in lymph node metastases was approximately as high as in the primary lesions of mucoepidermoid carcinoma. Only one patient with EGFR overexpression in the primary tumour had lower EGFR scores in the corresponding lymph node metastases. In a majority of the cases, the EGFR overexpression was retained in the metastases.

To our knowledge, the question of EGFR status in lymph node metastases versus primary mucoepidermoid carcinoma has yet to be addressed. Based on our results, we can draw the conclusion that the EGFR expression is stable when comparing the lymph node metastases with the primary mucoepidermoid carcinoma. It seems that the EGFR expression in the primary tumours, which can readily be determined after surgery or biopsy, can predict EGFR-positive metastases with a reasonably high probability.

The expression of HER2 in our samples was not so common as compared to that of EGFR. The overexpression of HER 2 was only found in two (4.3\%) out of the 46 cases of the primary mucoepidermoid carcinomas. A literature review shows an obvious controversy regarding the HER2 expression in mucoepidermoid carcinoma. Shrestha et al (26) and Sugano et al (27) found HER2 expression in only 4.5 
$(1 / 22)$ and $0 \%(0 / 9)$, respectively, of the studied mucoepidermoid carcinomas. Kernohan et al (28) reported positive immunostaining for HER 2 in only 1 out of 6 cases with mucoepidermoid carcinomas. However, Weed et al (23), Cho et al (24) and Press et al (25) reported HER2 overexpression in 25,36 and $38 \%$ of the studied cases. There may be many reasons for the observed differences, including patient selection, methodology of the immunohistochemical procedures, scoring and the definition of HER2 expression. For example, in the study of Press and colleagues (25), it was considered to be HER2 positive if any detectable staining could be found in a few scattered cells. Using the HercepTest scoring criterion, Glisson et al (22) reported a much lower frequency of HER2 positivity than the recent historical experience cited in the literature. The frequency of HER2 overexpression in the three most common subtype salivary malignancies (adenoid cystic, adenocarcinoma and mucoepidermoid) was only $8 \%$ (8/103).

No previous study can be found in the literature regarding the stability of the HER2 expression between primary mucoepidermoid carcinomas and the corresponding lymph node metastases. In our study, all the cases with lymph node metastases had a low HER2 expression (scored as 0 or $1+$ ) in the primary tumours. There was a reasonably good agreement between the HER2 expression in the primary tumours and the corresponding metastases, with just one case with $1+$ in the primary tumour changed to 0 in the corresponding lymph node. What is really important is the situation in the cases with HER2 overexpression. However, our results provided no further information in this respect.

Thus, the frequency of the HER2 overexpression in mucoepidermoid carcinoma seems to be low, which suggests a limited role of this receptor as a target in the treatment of mucoepidermoid carcinoma.

Additional strategies are needed to improve the survival and quality of life for patients with recurrent and disseminated mucoepidermoid carcinoma. Targeted therapy with antibodies or small-molecule tyrosine kinase inhibitors has proved to be a promising therapy strategy in lymphoma, lung, colorectal and breast cancer $(32,33)$ and may also be so for mucoepidermoid carcinoma. Furthermore, receptormediated tumour-targeted radionuclide therapy could be another strategy (11). This strategy is based on the delivery of therapeutically relevant radionuclides to disseminated tumour cells, hopefully with minimal damage to normal tissues.

For a receptor to be of interest for targeting, a similar expression in both the primary tumours and the disseminated lesions is required. The high frequency and stability in the EGFR expression in mucoepidermoid carcinoma is encouraging in the effort to use EGFR targeting agents (e.g. Iressa, Tarceva, Erbitux or radiolabelled antibodies) for the therapy of mucoepidermoid carcinoma, alone or in combination with radiotherapy and/or chemotherapy.

\section{Acknowledgements}

The authors acknowledge economical support from a grant from the National Natural Science Foundation of China to Q. Wei (No. 30470501).

\section{References}

1. Pinkston JA and Cole P: Incidence rates of salivary gland tumours: results from a population based study. Otolaryngol Head Neck Surg 120: 834-840, 1999.

2. Speight PM and Barrett AW: Salivary gland tumours. Oral Dis 8: 229-240, 2002.

3. Ito FA, Ito K, Vargas PA, de Almeida OP and Lopes MA: Salivary gland tumours in a Brazilian population: a retrospective study of 496 cases. Int J Oral Maxillofac Surg 34: 533-536, 2005.

4. Li LJ, Li Y, Wen YM, Liu H and Zhao HW: Clinical analysis of salivary gland tumour cases in West China in past 50 years. Oral Oncol (In press).

5. Emerick KS, Fabian RL and Deschler DG: Clinical presentation, management, and outcome of high-grade mucoepidermoid carcinoma of the parotid gland. Otolaryngol Head Neck Surg 136: 783-787, 2007.

6. Spiro RH: Management of malignant tumours of the salivary glands. Oncology 12: 671-680, 1998.

7. Bell RB, Dierks EJ, Homer L and Potter BE: Management and outcome of patients with malignant salivary gland tumours. J Oral Maxillofac Surg 63: 917-928, 2005.

8. Hicks MJ, el-Naggar AK, Flaitz CM, Luna MA and Batsakis JG: Histocytologic grading of mucoepidermoid carcinoma of major salivary glands in prognosis and survival: a clinicopathologic and flow cytometric investigation. Head Neck 17: 89-95, 1995.

9. Guzzo M, Andreola S, Sirizzotti G and Cantu G: Mucoepidermoid carcinoma of the salivary glands: clinicopathologic review of 108 patients treated at the National Cancer Institute of Milan. Ann Surg Oncol 9: 688-695, 2002.

10. Spiro RH, Huvos AG, Berk R and Strong EW: Mucoepidermoid carcinoma of salivary gland origin: a clinicopathologic study of 367 cases. Am J Surg 136: 461-468, 1978.

11. Carlsson J, Forssell Aronsson E, Hietala SO, Stigbrand T and Tennvall J: Tumour therapy with radionuclides: assessment of progress and problems. Radiother Oncol 66: 107-117, 2003.

12. Ciardiello F, Caputo R, Bianco R, Damiano V, Pomatico G, De Placido S, Bianco AR and Tortora G: Antitumour effect and potentiation of cytotoxic drugs activity in human cancer cells by ZD-1839 (Iressa), an epidermal growth factor receptorselective tyrosine kinase inhibitor. Clin Cancer Res 6: 20532063, 2000.

13. Hightower M: Erlotinib (OSI-774, Tarceva), a selective epidermal growth factor receptor tyrosine kinase inhibitor, in combination with chemotherapy for advanced non-small-cell lung cancer. Clin Lung Cancer 4: 336-338, 2003.

14. Goldberg RM: Cetuximab. Nat Rev Drug Discov: S10-S11, 2005.

15. Bonner JA, Harari PM, Giralt J, Azarnia N, Shin DM, Cohen RB, Jones CU, Sur R, Raben D, Jassem J, Ove R, Kies MS, Baselga J, Youssoufian H, Amellal N, Rowinsky EK and Ang KK: Radiotherapy plus cetuximab for squamous-cell carcinoma of the head and neck. N Engl J Med 354: 567-578, 2006.

16. Burtness B, Goldwasser MA, Flood W, Mattar B, Forastiere AA and Eastern Cooperative Oncology Group: Phase III randomized trial of cisplatin plus placebo compared with cisplatin plus cetuximab in metastatic/recurrent head and neck cancer: an Eastern Cooperative Oncology Group study. J Clin Oncol 23: 8646-8654, 2005.

17. Barnes DM and Miles DW: Response of metastatic breast cancer to trastuzumab? Lancet 355: 160-161, 2000.

18. Haddad R, Colevas AD, Krane JF, Cooper D, Glisson B, Amrein PC, Weeks L, Costello R and Posner M: Herceptin in patients with advanced or metastatic salivary gland carcinomas. A phase II study. Oral Oncol 39: 724-727, 2003.

19. Locati LD, Rinaldi G, Bossi P, Dagrada GP, Quattrone P, Cantu G and Licitra L: Herceptin plus chemotherapy in relapsed and/or metastatic salivary gland cancer. Oral Oncol 41: 97-98, 2005.

20. Yamada K, Iwai K, Okada Y and Mori M: Immunohistochemical expression of epidermal growth factor receptor in salivary gland tumours. Virchows Arch A Pathol Anat Histopathol 415: 523-531, 1989.

21. Gibbons MD, Manne U, Carroll WR, Peters GE, Weiss HL and Grizzle WE: Molecular differences in mucoepidermoid carcinoma and adenoid cystic carcinoma of the major salivary glands. Laryngoscope 111: 1373-1378, 2001. 
22. Glisson B, Colevas AD, Haddad R, Krane J, el-Naggar A, Kies M, Costello R, Summey C, Arquette M, Langer C, Amrein PC and Posner M: HER2 expression in salivary gland carcinomas: dependence on histological subtype. Clin Cancer Res 10: 944-946, 2004.

23. Weed DT, Gomez-Fernandez C, Pacheco J, Ruiz J, Hamilton-Nelson K, Arnold DJ, Civantos FJ, Zhang J, Yasin M, Goodwin WJ and Carraway KL: MUC4 and ERBB2 expression in major and minor salivary gland mucoepidermoid carcinoma. Head Neck 26: 353-364, 2004.

24. Cho KJ, Kim JY, Lee SS and Oh KK: Mucoepidermoid carcinoma of the salivary gland-a clinicopathologic and immunohistochemical study for c-erbB-2 oncoprotein. J Korean Med Sci 12: 499-504, 1997.

25. Press MF, Pike MC, Hung G, Zhou JY, Ma Y, George J, Dietz-Band J, James W, Slamon DJ, Batsakis JG and el-Naggar AK: Amplification and overexpression of HER-2/neu in carcinomas of the salivary gland: correlation with poor prognosis. Cancer Res 54: 5675-5682, 1994.

26. Shrestha P, Huang JW, Tsuji T, Shinozaki F, Maeda K, Sasaki K, Ueno K, Yamada K and Mori M: Rare expression of the c-erbB2 oncoprotein in salivary gland tumours: an immunohistochemical study. J Oral Pathol Med 21: 477-480, 1992.

27. Sugano S, Mukai K, Tsuda H, Hirohashi S, Furuya S, Shimosato Y, Ebihara S and Takeyama I: Immunohistochemical study of c-erbB-2 oncoprotein overexpression in human major salivary gland carcinoma: an indicator of aggressiveness. Laryngoscope 102: 923-927, 1992.
28. Kernohan NM, Blessing K, King G, Corbett IP and Miller ID Expression of c-erbB-2 oncoprotein in salivary gland tumours: an immunohistochemical study. J Pathol 163: 77-80, 1991.

29. Wei Q, Chen L, Sheng L, Nordgen H, Wester K, Carlsson J: EGFR, HER 2 and HER3 expression in esophageal primary tumours and corresponding metastases. Int J Oncol 31: 493-499, 2007.

30. Bilous M, Dowsett M, Hanna W, Isola J, Lebeau A, Moreno A, Penault-Llorca F, Rüschoff J, Tomasic G and van de Vijver M: Current perspectives on HER2 testing: a review of National Testing Guidelines. Mod Pathol 16: 173-182, 2003.

31. Mrhalova M, Plzak J, Betka J and Kodet R: Epidermal growth factor receptor-its expression and copy numbers of EGFR gene in patients with head and neck squamous cell carcinomas. Neoplasma 52: 338-343, 2005.

32. Mendelsohn J and Baselga J: Epidermal growth factor receptor targeting in cancer. Semin Oncol 33: 369-385, 2006.

33. Piccart-Gebhart MJ, Procter M, Leyland-Jones B, Goldhirsch A, Untch M, Smith I, Gianni L, Baselga J, Bell R, Jackisch C, Cameron D, Dowsett M, Barrios CH, Steger G, Huang CS, Andersson M, Inbar M, Lichinitser M, Lang I, Nitz U, Iwata H, Thomssen C, Lohrisch C, Suter TM, Ruschoff J, Suto T, Greatorex V, Ward C, Straehle C, McFadden E, Dolci MS, Gelber RD and Herceptin Adjuvant (HERA) Trial Study Team: Trastuzumab after adjuvant chemotherapy in HER2-positive breast cancer. N Engl J Med 353: 1659-1672, 2005. 